\title{
GROUPWISE NON-RIGID REGISTRATION ON MULTIPARAMETRIC ABDOMINAL DWI ACQUISITIONS FOR ROBUST ADC ESTIMATION: COMPARISON WITH PAIRWISE APPROACHES AND DIFFERENT MULTIMODAL METRICS
}

\author{
S. Sanz-Estébanez, Ó. Peña-Nogales, R. de Luis-García, S. Aja-Fernández and C. Alberola-López \\ LPI, ETSI Telecomunicación, Universidad de Valladolid, Spain
}

\begin{abstract}
Registration of diffusion weighted datasets remains a challenging task in the process of quantifying diffusion indexes. Respiratory and cardiac motion, as well as echo-planar characteristic geometric distortions, may greatly limit accuracy on parameter estimation, specially for the liver. This work proposes a methodology for the non-rigid registration of multiparametric abdominal diffusion weighted imaging by using different well-known metrics under the groupwise paradigm. A three-stage validation of the methodology is carried out on a computational diffusion phantom, a watery solution phantom and a set of voluntary patients. Diffusion estimation accuracy has been directly calculated on the computational phantom and indirectly by means of a residual analysis on the real data. On the other hand, effectiveness in distortion correction has been measured on the phantom. Results have shown statistical significant improvements compared to pairwise registration being able to cope with elastic deformations.
\end{abstract}

Index Terms - Non-rigid registration, Groupwise schemes, Multimodal metric, Diffusion-weighted magnetic resonance imaging, Distortion correction, Motion compensation

\section{INTRODUCTION}

Diffusion is described as the thermally induced behaviour of molecules moving in a microscopic random pattern, often referred to as Brownian motion. Diffusion-weighted magnetic resonance imaging (DW-MRI) is sensitive to this microscopic motion. Common approaches to estimate this motion assume a monoexponential decay in the DWI signal, providing characteristic quantitative parameters of the tissue, such as the well-known apparent diffusion coefficient (ADC). ADC has been shown to be a positive indicator to tumor response [1] due to its ability to measure displacement of water molecules, giving evidences about cellular organization and cell permeability of micro-structures [2].

However, in order to thoroughly validate these parameters as biomarkers, a robust parameter estimation methodology is mandatory, which is a complicated task, as an increasingly signal intensity dropout is observed when applying greater strength of the magnetic diffusion gradient values (the so-called $b$-values), as illustrated in Figure 1. Currently, simple estimators, such as the linear-least-squares method [3] are customary for ADC estimation. However, ADC estimation may also be greatly affected by several confounding factors, especially in a visceral organ like the liver, which exhibits considerable movement during imaging caused by respiratory [4] and cardiac motion [5]. In addition, ultrafast sequences typically used for diffusion studies, i.e. echo planar imaging (EPI), suffer from geometric distortions as well as local signal dropouts caused by static magnetic field inhomogeneities [6]. The resultant distortions are commonly seen near tissue interfaces, where magnetic susceptibility changes rapidly.

Denoising schemes [7] have been proposed to robustify ADC estimation; in our case, we will focus on multiparametric (multiple $b$-values) abdominal DWI registration schemes to alleviate the effects of these confounding factors. Most approaches pose the registration problem from a pairwise standpoint [8] using an [ideally] undistorted image as reference; this procedure, however, is prone to an undesired bias towards the a priori chosen template [9], which, depending on its quality, may give rise to multiple outliers in the alignment. On the other hand, groupwise approaches are based on an image reference that is built out of the whole image set to be registered, so that the template bias disappears.

Methodologies for multiparametric registration towards robust ADC estimation have been designed based on distortion correction methods [10] or pairwise connection metrics [11]. Groupwise approaches have been addressed in [12] using prior

This work was partially supported by the Spanish Ministerio de Ciencia e Innovación under Research Grant TEC2013-44194-P, the European Regional Development Fund (ERDF-FEDER) under Research Grant TEC2014-57428-R and the Spanish Junta de Castilla y León under Grant VA069U16. 

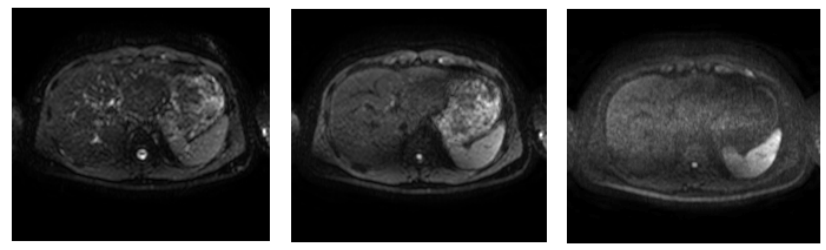

Fig. 1. Axial slices of DWI acquisition in a healthy patient for $b$-values of 0,100 and $1000 \mathrm{~s} / \mathrm{mm}^{2}$ (from left to right).

structure segmentations or by minimizing the regression fitting error [13]. However, none of them explicitly take into account interaction of liver motion artifacts and EPI geometrical distortions on the deformation field jointly.

In this paper, we address the importance of a registration scheme in the robustness analysis of multiparametric abdominal DWI acquisitions by assessing the adequateness of groupwise and pairwise paradigms within an elastic transformation model as well as the suitability of different multimodal voxel-based metrics.

\section{MATERIALS AND METHODS}

\subsection{Materials}

For the validation of the proposed scheme we have resorted to a three-fold procedure. To begin with, (1) a synthetic experiment has been carried out using a simulation environment based on the 4D extended cardio-torso (XCAT) phantom [14]. The phantom consists of a whole body model that contains high level detailed anatomical labels, which feed a high resolution image synthesis procedure. The 4D XCAT phantom incorporates state-of-the-art respiratory and cardiac mechanics, which provide sufficient flexibility to simulate cardio-torso motion from a user-defined parameter set. Although we assume breath-hold acquisitions, different inspiration levels are simulated for different $b$-values. Residual cardiac artifacts have been neglected. A synthetic deformation field is added in order to simulate typical geometric distortions of EPI acquisitions. Therefore, the phantom provides us not only with the images themselves, but also with a ground-truth ADC map.

Additionally, we have performed two MRI acquisitions, first, (2) on a phantom consisting of a bottle filled with a watery solution and finally (3) with a sample of four healthy volunteers. Axial SENSitivity Encoding (SENSE) DWI and T2 weighted (T2w) Turbo Spin Echo sequences have been acquired on a Philips Achieva 3T scanner in each case of study. The latter will be used to manually delineate the whole liver as region of interest (ROI) on which meaningful measurements will be provided. Acquisition and resolution details for these sequences are shown in Table 1.

\subsection{Methods}

The proposed method has been applied to the groupwise registration of different $b$-value images in axial abdominal DWI acquisitions and, specifically, for robust estimation of diffusion parameters on both lobes of the liver.

The [ideal] underlying monoexponential decay model applied to the images can be described as follows:

$$
S(b)=S_{0} e^{-b \cdot \mathrm{ADC}}
$$

where $S(b)$ and $S_{0}$ denote the signal intensity obtained with the diffusion gradient $b$-value of $b$ and null. Sampling of $b$ values is finer for lower $b$-values according to [15]. Nine of this $b$-values are common for every sequence. Those are $b \in$ $\{0,10,20,50,150,300,500,800,1000\} \mathrm{s} / \mathrm{mm}^{2}$. Finally, ADC has been estimated by the linear-least-squares method.

For the registration scheme, the local transformation $T$ is represented as a combination of B-spline [16] FFDs. A gradientdescent/ascent optimization scheme is performed for the optimization of the registration metric $H$.

The developed multimodal metrics for the groupwise registration approach are the following:

- Entropy of the distribution of intensities (EDI) [17]:

$$
H(\mathbf{x})=S_{\mathcal{T}}(\mathcal{I}(\mathcal{T}(\mathbf{x}))) \simeq \frac{-1}{N} \sum_{n=1}^{N} \log \left(p\left(I_{n}\left(T_{n}(\mathbf{x})\right)\right)\right)
$$

where $p\left(I_{n}\left(T_{n}(\mathbf{x})\right)\right)$ is a Parzen window estimation [18] of the intensity distribution of corresponding pixels. This metric favours those solutions in which pixel intensities are well concentrated in the intensity space. 


\begin{tabular}{|r|r|r|r|}
\hline Params. & XCAT & DWI phant. & DWI volunt. \\
\hline$\Delta_{p}$ & 1 & 0.7145 & $1.23-1.85$ \\
\hline$\Delta_{l}$ & 10 & 5.5 & 5.5 \\
\hline$N_{p}$ & 308 & 480 & $224-320$ \\
\hline$N_{b}$ & 9 & 12 & $9-20$ \\
\hline$N_{s}$ & 5 & 12 & $20-40$ \\
\hline$T_{E}$ & 65 & 31.96 & $65.65-72.1$ \\
\hline$N_{g}$ & 1 & 3 & 3 \\
\hline Card. & 1 & N/a & $\sim 1$ \\
\hline Resp. & 5 & N/a & Free \\
\hline
\end{tabular}

Table 1. Details on the image sequences used in the paper. $\Delta_{p}$ : Spatial Resolution (mm). $\Delta_{l}:$ Slice Thickness (mm). $N_{p}$ : Number of pixels along each direction. $N_{b}$ : Number of $b$-values. $N_{s}$ : Number of slices. $T_{E}$ : Echo Time (ms). $N_{g}$ : Number of diffusion gradients. Card.: Cardiac Period (s). Resp.: Respiratory Period (s).

- Variance of the local entropy (VLE): for the groupwise approach we will assume that this local entropy is preserved across the image set. Hence, the voxelwise metric will be considered as the sum of squared differences of the local entropy images $S_{\mathcal{N}}$ obtained from the transformed image $I$ as described in [19] as:

$$
S_{\mathcal{N}}(\mathbf{I}(\mathcal{N}(\mathbf{x})))=\frac{-1}{|\mathcal{N}|} \sum_{\mathbf{x}^{\prime} \in \mathcal{N}(\mathbf{x})} p\left(I\left(\mathbf{x}^{\prime}\right)\right) \log \left(p\left(I\left(\mathbf{x}^{\prime}\right)\right)\right)
$$

with $|\mathcal{N}|$ the cardinality of the neighbourhood whose radius has been set empirically to 6 pixels.

- Normalized cross-correlation (NCC):

$$
H(\mathbf{x})=\frac{1}{N} \sum_{n=1}^{N} \frac{<\overline{I_{n}\left(T_{n}(\mathcal{P}(\mathbf{x}))\right)}, \overline{\mu((\mathcal{P}(\mathbf{x}))}>^{2}}{<\overline{I_{n}\left(T_{n}(\mathcal{P}(\mathbf{x}))\right)}><\overline{\mu(\mathcal{P}(\mathbf{x}))}>}
$$

where $\mu(\mathbf{x})=\frac{1}{N} \sum_{n=1}^{N} I_{n}\left(T_{n}(\mathbf{x})\right)$, while $\overline{I_{n}\left(T_{n}(\mathcal{P}(\mathbf{x}))\right)}$ corresponds to the image intensities in a patch $\mathcal{P}$ with its spatial mean subtracted and $<,>$ to the inner product defined in [20]. NCC depends only on estimates of mean and variance from samples of the given patch.

- Modality independent neighbourhood descriptor (MIND): an image descriptor, built from within-patch distances $D_{p}$ and variance estimates $V$ on a six-neighbourhood search region $R$, defined as:

$$
\operatorname{MIND}(I, \mathbf{x}, r) \propto \exp \left(\frac{D_{p}(I, \mathbf{x}, \mathbf{x}+r)}{V(I, \mathbf{x})}\right) .
$$

with $r \in R$. Afterwards, sum of simple monomodal similarity measures built from MIND differences is used as a metric, as described in [21]. 


\section{RESULTS}

In this section, we test the ability of the proposed methods for motion compensation, distortion correction and robust ADC estimation. We have carried out a synthetic experiment with the data provided by the XCAT computational phantom, manually distorted thereafter, on which we have measured the absolute error (over the previously defined ROI) on ADC estimation for the groupwise multimodal metrics of section 2.2 and its counterpart for the pairwise case. In the latter, the image template is taken from the T2w sequence. Notice that this image is free of EPI distortions, so it is favorable case for pairwise approaches.

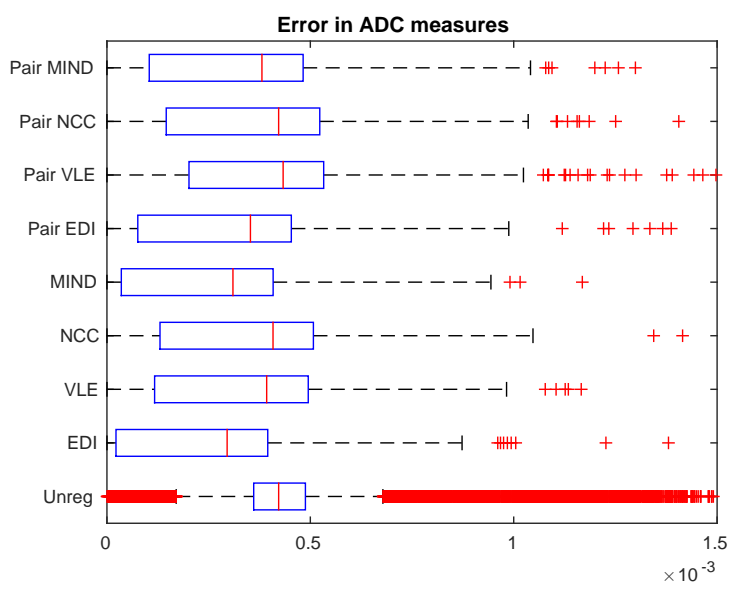

Fig. 2. Error on ADC estimation for proposed metrics

In Figure 2 we show the boxplot diagrams of the absolute error distributions on ADC estimation as a measure of accuracy in motion compensation and distortion correction. Presence of outliers is greatly diminished when performing previous registration.

Mann-Whitney U-tests were performed for each pair of error distributions. Significant differences have been found for EDI, MIND and VLE metrics between its groupwise and pairwise approaches (every metric holds $p<10^{-3}$ ). Significant differences also exist within groupwise metrics, with the exception of EDI and MIND metrics $(p=0.35)$, that exhibit best, albeit similar performance in terms of error, specially when compared to the original data ( $p<10^{-9}$ for both).

For the real data, first, we have tested the ability of the aforementioned groupwise metrics to correct EPI distortions on the MRI phantom described in section 2.1. We have performed a quantitative analysis of the overlapping (using the Dice coefficient) between foregrounds obtained from the registered DWI and the undistorted T2w sequences.

In Figure 3 we show boxplot diagrams of the Dice coefficient distributions for each groupwise and pairwise metric and the original data. No significant differences are found for Mann-Whitney U-tests over Dice coefficient distributions of groupwise metrics compared to its pairwise counterpart.

Aditionally, Kruskal-Wallis tests were performed, finding significant differences within groupwise metrics $(p=0.0027)$ and with the original unregistered results $\left(p<10^{-6}\right)$. VLE and MIND metrics seem to have better recovered from EPI distortion in this scenario.

In addition, for the volunteer data, as a ground-truth is not available, we have resorted to a goodness-of-fit analysis showing the discrepancy between the data and the diffusion model in equation (1). We have measured the residual sum of squares (RSS) obtained in the ADC estimation for each groupwise and pairwise metric as a measure of robustness.

After performing U-tests, no differences were found for RSS distributions neither between groupwise or pairwise metrics nor with the original data. In this case, apparently, the acquisition protocol parameters and the estimation model have had a greater impact on the RSS than the alignment itself.

\section{CONCLUSIONS}

In this paper, we have proposed a non-rigid framework for the motion compensation in multiparametric abdominal DWI acquisitions. The groupwise approach helps to cope with changes in signal intensity and corrects for geometrical distortions. This methodology has been applied to the alignment of DWI sequences in the liver, showing significant improvements in performance compared to pairwise approaches. 


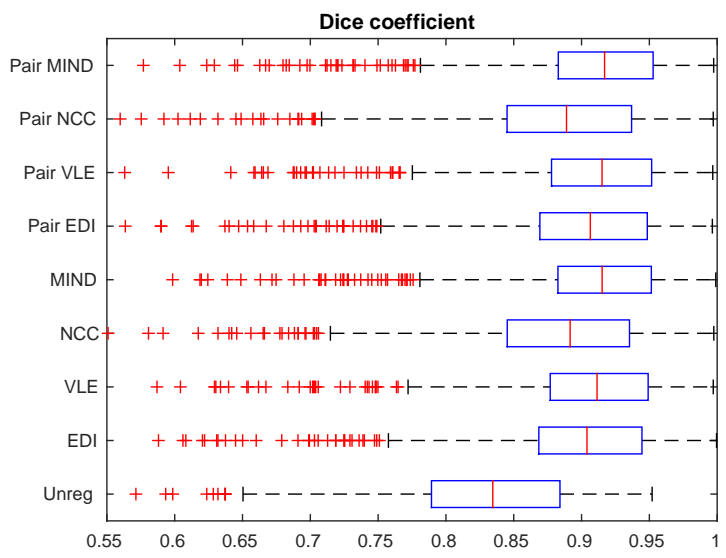

Fig. 3. Boxplot diagrams of Dice Coefficient of foregrounds obtained from registered DWI and T2w sequences

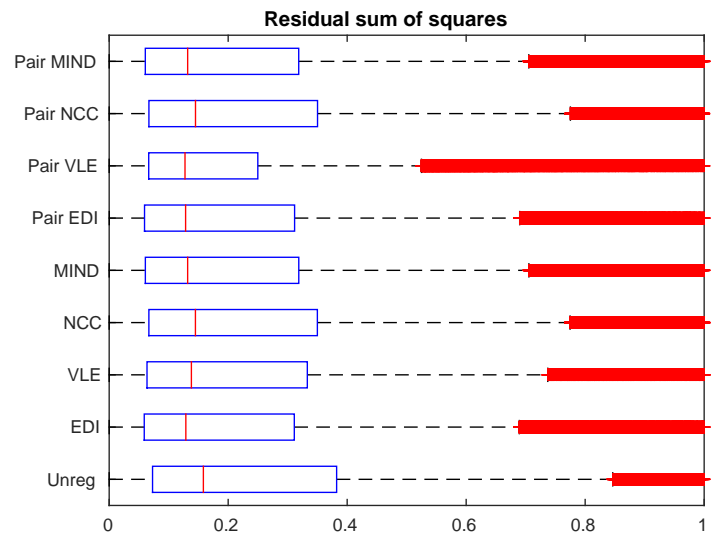

Fig. 4. Distribution of RSS obtained from the ADC estimation for each registration metric

The metric choice is also an important issue for avoiding outliers and making the registration process more robust. Thus, although the EDI metric is ranked first in terms of error and RSS, it seems more prone to the presence of outliers than other tested metrics, especially MIND. Consequently, we do not have enough evidence to support one metric over the other for this particular problem.

\section{REFERENCES}

[1] T. Kim, T. Murakami, S. Takahashi, M. Hori, K. Tsuda, and H. Nkamura, "Diffusion-weighted single-shot echoplanar MR imaging for liver disease," Amer. Journ. Roent., vol. 173, pp. 393-398, 1999.

[2] D. Le Bihan, "Diffusion MRI: what water tells us about the brain," EMBO Molecular Medicine, vol. 6, no. 5, pp. 569-573, 2014.

[3] S. Heiland, O. Dietrich, and K. Sartor, "Diffusion-weighted imaging of the brain: comparison of stimulated- and spin-echo echo-planar sequences," Neurorad., vol. 43, no. 6, pp. 442-447, 2001.

[4] Y. Mazaheri, RKG. Do, A. Shukla-Dave, JO. Deasy, YM. Lu, and O. Akin, "Motion Correction of Multi-b-value Diffusion-weighted Imaging in the Liver," Acad. Radiol., vol. 19, no. 12, pp. 1573-1580, 2012.

[5] J. Liau, J. Lee, ME. Schroeder, CB. Sirlin, and M. Bydder, "Cardiac Motion in Diffusion Weighted MRI of the Liver: Artifact and a Method of Correction," J. Magn. Reson. Imaging., vol. 35, no. 2, pp. 318-327, 2012. 
[6] MA. Bernstein, KF. King, and XJ. Zhou, Handbook of MRI pulse sequences, Burlington, MA: Elsevier Academic Press, 2004.

[7] X. Zhang, G. Hou, JA. Ma, W. Yang, B. Lin, and Y. Xu, "Denoising MR Images Using Non-Local Means Filter with Combined Patch and Pixel Similarity," PLoS ONE, vol. 9, no. 6, pp. 1-12, 2014.

[8] M. Wu, LC. Chang, L. Walker, H. Lemaitre, AS. Barnett, S. Marenco, and C. Pierpaoli, "Comparison of EPI Distortion Correction Methods in Diffusion Tensor MRI Using a Novel Framework,” in MICCAI, 2008, vol. 5242, pp. 321-329.

[9] C. Wachinger and N. Navab, "Simultaneous Registration of Multiple Images: Similarity Metrics and Efficient Optimization,” IEEE Trans. Patt. Anal. Mach. Int., vol. 35, no. 5, pp. 1221-1233, 2013.

[10] X. Hong, X. Vinh, I. Teh, JR. Soh, and KH. Chuang, "Evaluation of EPI distortion correction methods for quantitative MRI of the brain at high magnetic field," Megn. Reson. Med., vol. 33, pp. 10981105, 2015.

[11] JM. Guyader, L. Bernandin, NHM. Douglas, DHJ. Poot, WJ. Niessen, and S. Klein, "Influence of image registration on apparent diffusion coefficient images computed from freebreathing diffusion mr images of the abdomen," J. Magn. Reson. Imaging., vol. 42, no. 2, pp. 315-330, 2015.

[12] H. Veeranghavan, RKG. Do, DL. Reidy, and JO. Deasy, "Simultaneous segmentation and iterative registration method for computing adc with reduced artifacts from dw-mri," Med. Phys., vol. 42, no. 5, pp. 2249-2260, 2015.

[13] EN. Kornapoulos, EI. Zacharaki, P. Zerbib, C. Lin, A. Rahmouni, and N. Paragios, "Deformable group-wise registration using a physiological model: application to Diffusion-Weighted MRI," in ICIP, Arizona, United States, 2016.

[14] WP. Segars, G. Sturgeon, S. Mendonca, J. Grimes, and BMW. Tsui, "4D XCAT phantom for multimodality imaging research," Medical Physics, vol. 37, pp. 4902-4915, 2010.

[15] A. Luna, R. Ribes, and JA. Soto, Diffusion MRI outside the brain, Springer-Verlag, 2012.

[16] D. Rueckert, P. Aljabar, RA. Heckemann, JV. Hajnal, and A. Hammers, " Diffeomorphic Registration using B-Splines," in MICCAI, 2006, vol. 4191, pp. 702-709.

[17] EG. Learned-Miller, "Data Driven Image Models through Continuous Joint Alignment," IEEE. Trans. Patt. Anal. and Mach. Intel., vol. 28, pp. 236-250, 2006.

[18] WM. Wells III, P. Viola, H. Atsumi, S. Nakajima, and R. Kikinis, "Multi-Modal Volume Registration by Maximization of Mutual Information," Med. Image Anal., vol. 1, pp. 35-51, 1996.

[19] C. Wachinger and N. Navab, "Entropy and Laplacian Images: Structural Representations for Multi-Modal Registration," Med. Im. Anal., vol. 16, pp. 1-17, 2012.

[20] BB. Avants, CL. Epstein, M. Grossman, and JC. Geel, "Symmetric Diffeomorphic Image Registration with CrossCorrelation: Evaluating Automated Labeling of Elderly and Neurodegenerative Brain," Med. Image Anal., vol. 12, pp. 26-41, 2008.

[21] MP. Heinrich, M. Jenkinson, M. Bhushnan, T. Matin, FV. Gleeson, M. Brady, and JA. Schnabel, "MIND: Modality independent neighbourhood descriptor for multi-modal deformable registration," Med. Image Anal., vol. 16, pp. 1423$1435,2012$. 